\title{
Autophagy regulation by microRNAs in chemotherapy resistance (Review)
}

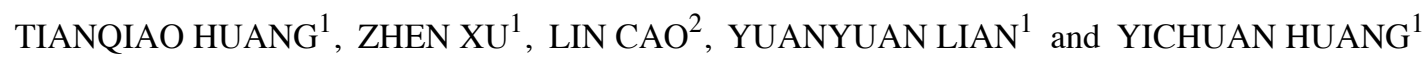 \\ ${ }^{1}$ Department of Otolaryngology, The Affiliated Hospital of Qingdao University, \\ Qingdao, Shandong 266000; ${ }^{2}$ Department of Otolaryngology Head and Neck Surgery, \\ Beijing Tongren Hospital, Affiliated to Capital Medical University, Beijing 100730, P.R. China
}

Received January 27, 2020; Accepted June 4, 2020

DOI: $10.3892 /$ or.2020.7655

\begin{abstract}
Chemotherapy, as an adjuvant therapy, utilizes drugs to treat cancer, and resistance to therapeutic drugs limits the efficacy of chemotherapy treatments. Several mechanisms have been proposed to improve the effect of tumor chemotherapy in order to overcome drug resistance. Among these, autophagy mediated by microRNAs (miRNAs) is one of the primary mechanisms. A large number of molecules targeted by miRNAs are involved in each step of the autophagic pathway. Recent advancement in chemotherapy research has revealed that miRNAs involved in the autophagy process target some of these molecules, thereby influencing the therapeutic effect of chemotherapy drugs. Thus, miRNAs appear to be potential tools or targets with which to suppress tumor growth and should be studied in further details for their clinical application against drug resistance.
\end{abstract}

\section{Contents}

1. Introduction

2. miRNA-mediated autophagy changes with respect to drug resistance in tumor treatment

3. Conclusion and future directions

\section{Introduction}

Chemotherapy, as the first-line of treatment or adjuvant treatment for tumors, often delivers mixed outcomes (1). The primary reason for this is drug resistance encountered during chemotherapy. Overcoming drug resistance by tumors is a basic and challenging initiative to resolve the insensitivity of

Correspondence to: Dr Yichuan Huang, Department of Otolaryngology, The Affiliated Hospital of Qingdao University, 16 Jiangsu Road, Qingdao, Shandong 266000, P.R. China

E-mail: huangyichuanqd@163.com

Key words: cancer, autophagy, miRNA, chemotherapy resistance tumors toward chemotherapy. Recent studies have focused on microRNAs (miRNAs) as a tool to suppress tumorigenesis, which is expected to assist in the development of effective chemotherapy drugs (2).

miRNAs are endogenous non-coding RNAs with an approximate length of 23 nucleotides, which can regulate the expression of target genes via interaction with its 3'-untranslated regions (3). miRNAs are involved in diverse biological and pathological processes, including the generation and development of cancer. They also act on target genes to inhibit tumor resistance to chemotherapy drugs (4). Various known mechanisms of miRNA regulation include the overexpression of multidrug resistance (MDR) transporters, defects in cell-cycle and the apoptotic machinery, induction of autophagy, alternation of anticancer drug metabolism, alteration in drug targets, DNA repair, and disruption of redox homeostasis. Among these, autophagy regulation is one of the most critical mechanisms (5) that demonstrates an important mechanism to overcome chemotherapy resistance of various tumors (6).

In 2016, Yoshinori Ohsumi won the Nobel Prize in physiology or medicine in recognition of his outstanding achievements in emphasizing the importance of autophagy as the key player in understanding human diseases. Autophagy is the quality-control mechanism of a cell to regulate its cellular homeostasis (7). It plays a dual role in tumorigenesis, either as a promoter or suppressor of tumors (8). Recent studies, however, have focused on the protective effect of autophagy on tumor cells and have further strengthened the belief that autophagy can promote apoptosis of tumor cells and inhibit their proliferation (6). A large number of molecules are involved in each step of the autophagic pathway (6,9-11) (Fig. 1), and, during this process, miRNAs target some of these molecules and thereby participate in the generation of tumor chemotherapy resistance.

The present review summarizes some of the relevant works in recent years and their perspective on the role of miRNA-mediated autophagy in tumor chemotherapy resistance.

2. miRNA-mediated autophagy changes with respect to drug resistance in tumor treatment

miRNA-mediated autophagy initiation changes the pattern of drug resistance in tumors. Autophagy initiation involves 
2 major complexes: The UNC-51-like autophagy-activating kinase (ULK)1 complex and the class III PI 3-kinase (PI3K) complex (12). Some miRNAs target any one or more of the above-mentioned 2 compounds or their inhibitors and trigger autophagy alteration, thereby changing the tumor drug resistance pattern (Table I).

The ULK1 complex includes ULK1, autophagy-related gene (ATG)13 protein, focal adhesion kinase family interacting protein of $200 \mathrm{kDa}$ (FIP200) and ATG101 in humans. ULK1 is a part of a family of ULK1-4 kinases that play an important role in autophagy (12). ULK1 is targeted by miRNA-26a/b, and the in vitro overexpression of ULK1 promotes apoptosis by inhibiting autophagy; moreover, the overexpression of miRNA-26a/b has been shown to enhance the sensitivity of hepatocellular carcinoma cells (HCC) to doxorubicin in vivo in xenograft models of nude mice (13). In another report, ULK1-mediated autophagy protected lung adenocarcinoma cells from the toxic effects of tyrosine kinase inhibitors (TKIs), and high expression of miR-106a was found to inhibit $U L K 1$ expression, reduce autophagy, and increase the cytotoxic effects of TKIs (14). Another study showed that isoliquiritugenin (ISL), a natural flavonoid, possesses anticancer properties and that it could inhibit the expression of miR-25, leading to the upregulation of its target gene $U L K 1$ and an increase in the extent of autophagy. These events ultimately led to the accelerated degradation of ATP-binding cassette sub-family $\mathrm{G}$ member 2 (ABCG2) through the autophagy-lysosomal pathway, resulting in improved toxicity of chemotherapy drugs against breast cancer cells (15). FIP200, a large $U L K 1$-interacting protein, has a predicted coiled-coil protein involved in scaffolding (16). Cheng et al reported that the expression of miR-409-3p was enhanced in cisplatin-sensitive cells when compared with cisplatin-resistant cells, and the overexpression of miR-409-3p decreased the expression of FIP200 and the related autophagy, thereby increasing the cisplatin sensitivity of ovarian cancer cells (17).

The PI3K complex occurs downstream of ULK1. The PI3K complex consists of autophagy and Beclin-1 regulator 1 (AMBRA1), Beclin-1, vacuolar protein sorting 34 (VPS34) (phosphatidylinositol), VPS15, UV resistance-associated gene (UVRAG) and ATG14L. ATG14L is a Beclin-1-associated autophagy key regulator (18). IncRNAs are non-coding RNA with a length of more than 200 nucleotides. IncRNA lacks the coding ability, but it is a functional molecule (19) with multiple effects on miRNAs (20). An IncRNA can be used as an miRNA sponge as it competes with miRNA to bind to the target mRNA, and some lncRNAs can also be converted into miRNAs. It was found that knockdown of Lnc0515 induced the overexpression of miR-140-5p, which targets ATG14, one of the components of the PI3K complex. This event eventually caused inhibition of autophagy, thereby alleviating the chemoresistance of myeloma cells (18). ATG14 is also known to be targeted by miR-152, while the transcription factor early growth response 1 (EGRI) regulates miR-152 upstream, the overexpression of either EGR1 or miR-152 led to the inhibition of ATG14 expression as well as the inhibition of autophagy, eventually increasing the cisplatin toxicity to ovarian cancer cells and thereby weakening drug resistance (21). Luciferase reporter assay identified that several miRNAs, such as miR-30a, which directly binds to the 3'-UTR of Beclin-1, inhibited
Beclin-1 expression. When miR-30a was overexpressed, Beclin-1-mediated autophagy was inhibited, which not only promoted the chemotherapy-induced apoptosis of osteosarcoma cells but also enhanced the toxicity of sorafenib to renal cell carcinoma (RCC) cells $(22,23)$. The sensitivity of undifferentiated thyroid carcinoma (UTC) to cisplatin is heterogeneous, which may be related to the downregulation of miR-30d; both in vivo and in vitro experiments confirmed that the downregulation of miR-30d could target and promote Beclin-1 expression, leading to an increase in autophagy and in the insensitivity of UTC cells to cisplatin (24). miR-216b was found to target Beclin-1, UVRAG, and ATG5, 3 essential autophagy genes, directly to weaken autophagy and enhance the antitumor activity of the drug vemurafenib in BRAF (V600E) melanoma cells (25). In addition, Tan et al reported that the overexpression of miR-409-3p inhibited Beclin-1 expression and autophagy, and thus enhanced the chemosensitivity of colon cancer cells to the drug oxaliplatin in vivo and in vitro (26). Other research also noted a reduction in the expression level of miR-216b in paclitaxel-treated non-small cell lung carcinoma (NSCLC) cells. As the expression level of miR-216b increased, the Beclin-1 mRNA translation level decreased, autophagy was inhibited, and the toxicity of paclitaxel to NSCLC was enhanced (27). High-mobility group box 1 (HMGB1) is a type of non-histone protein that has been widely reported to play a key role in the induction of autophagy. Several studies have suggested that HMGB1 binds to the PI3K complex to facilitate autophagic progression (28-33). miR-142-3p was found to directly downregulate $H M G B 1$, inhibit autophagy induced by anticancer drugs, and increase the chemosensitivity of NSCLC in both in vitro and in vivo experiments (29). In vivo and in vitro experiments showed that the expression of miR-410-3p was downregulated in tumor cells and in transplanted tumor tissues with chemotherapy-resistance to gemcitabine; after intervention, high expression of miR-410-3p was targeted to inhibit $H M G B 1$ and its induced autophagy, which resulted in enhanced sensitivity of chemotherapy (30). HMGBI is also the target gene of miR-218, which binds to the 3'-UTR of this gene, inhibits the expression of the target gene, inhibits autophagy, and was found to improve the sensitivity of endometrial cancer cells to paclitaxel chemotherapy (31). Similarly, miR-22 and miR-34a were also found to target $H M G B 1$ and inhibit its expression, inhibit autophagy, and restore the sensitivity of osteosarcoma and retinoblastoma cancer cells to chemotherapy drugs $(32,33)$.

mTOR, a serine/threonine kinase, is a master regulator of cellular metabolism and the key regulator of autophagy. It forms 2 complexes: mTORC1 and mTORC2; the former inhibits autophagy-initiation by preventing the formation of ULK1 and PI3K complexes (34). miR-338-3p was found to increase autophagy by downregulating the expression of its target $m T O R$, leading to the resistance of colon cancer cells containing P53 mutants to 5-fluorouracil (35). Downregulation of lncRNA-CASC2 in glioma resulted in an increase in the miR-193a-5p expression level and a decrease in the mTOR expression, which further induced protective autophagy, resulting in temozolomide resistance (36). The PI3K/PTEN/Akt/mTOR signaling pathway has been implicated in the resistance to chemoradiotherapy (37). Phosphatase and tension homolog (PTEN), which encodes a phosphatase 


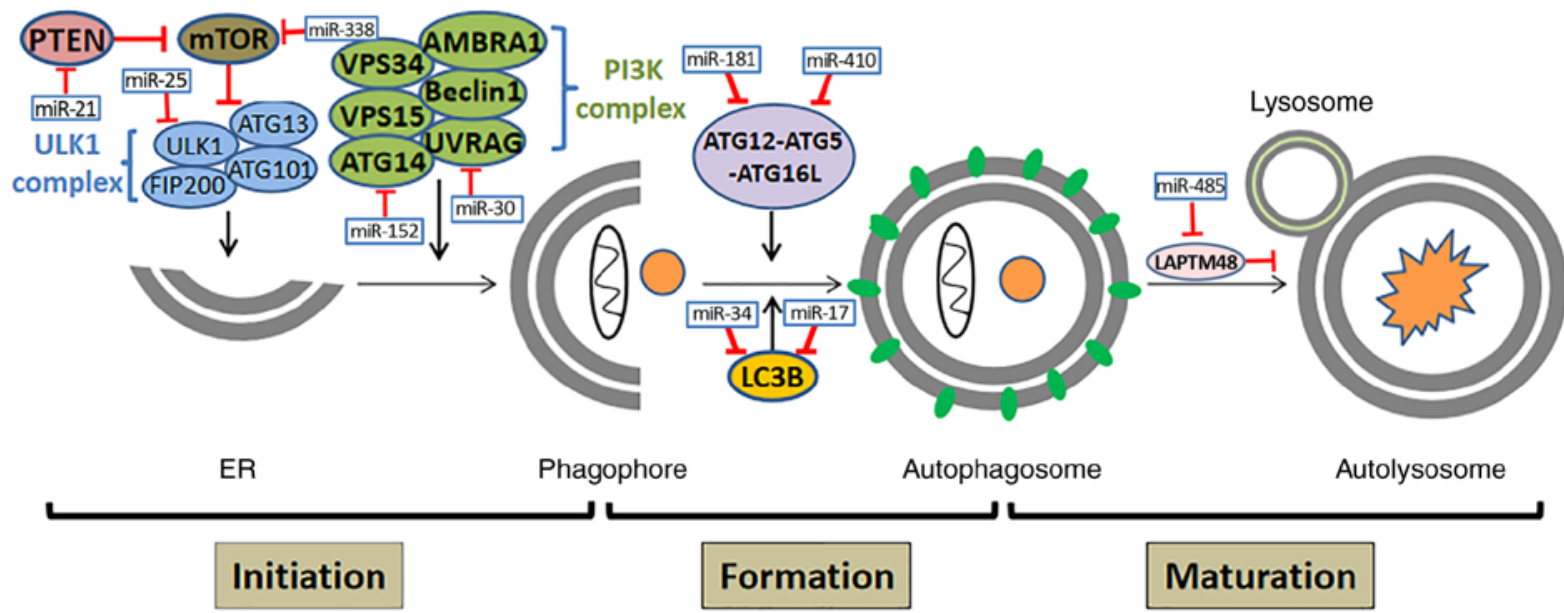

Figure 1. The molecules involved in each step of the autophagy process. Autophagy initiation depends on two vital complexes: The ULK1 complex (ULK1, FIP200, ATG13, ATG101) and PI3K complex (VPS34, VPS15, ATG14, AMBRA1, Beclin1, UVRAG). mTOR plays a suppressive role in the initiation of autophagy; autophagosome formation involves the formation of a complex of ATG12-ATG5-ATG16L and LC3B; other molecules participate in the maturation of the autolysosome. Some key miRNAs regulate their target genes to participate in every stage of autophagy. ULK1, UNC-51-like autophagy-activating kinase 1; PI3K, the class III PI 3-kinase; FIP20, focal adhesion kinase family interacting protein of $200 \mathrm{kDa}$; ATG, autophagy-related gene; ABCG2, ATP-binding cassette sub-family G member 2; VPS, vacuolar protein sorting; AMBRA1, autophagy and Beclin-1 regulator 1; UVRAG, UV resistance-associated gene; LC3B, microtubule-associated protein light chain 3; LAPTM4B, lysosome-associated protein transmembrane $4 \beta$; EGR1, early growth response 1; HMGB1, high-mobility group box 1; PTEN, phosphatase and tension homolog.

protein, is an essential tumor-suppressor gene. It activates the mTOR pathway and inhibits autophagy (38). In MG-63/ADM osteosarcoma cells, miR-155, p-AKT, and p-mTOR were found to be overexpressed. PTEN, the target gene of miR-155, is a positive regulator of autophagy, and the overexpression of miR-155 was found to inhibit PTEN and reduce autophagy induction, simultaneously. In addition, expression of $A K T$ and $m T O R$ was found to activate the $P I 3 K / A K T / m T O R$ signaling pathway and inhibit autophagy, which in turn led to increased adriamycin resistance in tumor cells (39). The knockdown of miR-21 expression in breast cancer cells was found to lead to an increase in the expression of PTEN and the inhibition of the activation of $A K T$. This event blocked the $P I 3 K / A K T / m T O R$ signaling pathway, increased autophagic cell death, and partially overcame the endocrine resistance of tumor cells to tamoxifen and fulvestrant (40). Another study reported that miR-21 expression was higher in HCC cells resistant to sorafenib, which resulted in decreased expression of PTEN, increased activation of the $A K T / m T O R$ pathway, and significant inhibition of autophagy (41).

miRNA-mediated autophagosome formation changes the pattern of drug resistance in tumors. The two essential complexes of autophagosomes are ATG12-ATG5-ATG16L and LC3B $(42,43)$. Other miRNAs target any one or more of the above-mentioned two compounds, trigger autophagy alteration, and change the response of the tumor to chemotherapy drugs (Table II). ATG5-ATG12 conjugation depends on ATG7 (an E1-like ubiquitin-activating enzyme) and ATG10 (an E2-like ubiquitin carrier protein), which pairs with ATG16L dimers to form the ATG5-ATG12-ATG16L complex; this complex associates with the outer membrane of the extending phagophore (10). By competitively inhibiting miR-23b-3p, lncRNA MALAT1 was found to increase the expression of $A T G 12$, which in turn led to increased autophagy and drug resistance in gastric cancer cells (44). miR-410 was found to reduce expression of $A T G 16 L 1$ by directly targeting its 3'-UTR, subsequently inhibiting autophagy and improving the response of osteosarcoma cells to chemotherapy drugs (rapamycin, adriamycin, and cisplatin) (45). ATG5 is an miR-181a target, and overexpression of miR-181a was found to inhibit ATG5 and increase the sensitivity of gastric cells SGC7901/CDDP to cisplatin in a mouse model, in which tumor xenografts were downsized (46). PU.1-miR-142-3p targets ATG5/ATG16L1. Upregulation of heterotopic miR-142-3p, was found to reduce sorafenib-induced autophagy, enhance sorafenib-induced apoptosis, and inhibit cell growth, enhancing HCC cell sensitivity to sorafenib (47). ATG12 and $H M G B 2$, target genes of miR-23b-3p, are essential elements of autophagy. Following miR-23b-3p overexpression, the expression and ATG12 and $H M G B 2$-mediated autophagy are inhibited, and as a result, the sensitivity of gastric cancer cells to chemotherapy drugs is increased (48).

Microtubule-associated protein light chain 3 (LC3B) is another ubiquitin-like system involved in autophagosome formation. LC3B is encoded by ATG8 and it is a product of the cleavage of ProLC3 by ATG4 and ATG7. One previous study showed that hypermethylation causes downregulation of miR-34a, which results in the upregulation of miR-34. Demethylated miR-34a targets $A T G 4 B$, thereby downregulating $A T G 4 B$-induced autophagy by activating the $A M P K / m T O R$ pathway, with an increased sensitivity to chemotherapy in prostate cancer (PCa) cells (49). THP (pirarubicin) treatment significantly reduced the size of the transplanted tumor. In vivo experiments revealed that the overexpression of miR-34c-5p in cells led to a decrease in the inhibition of autophagy by $A T G 4 B$, which increased the sensitivity to THP (50). Several studies have shown that multiple miRNAs target ATG7 to mediate autophagy in order to alter the pattern of drug resistance in tumors. For example, MTT assay demonstrated that dual treatment of inhibitors of abelson non-receptor tyrosine kinase (c-ABL) (imatinib) and EGFR (lapatinib) suppressed 
Table I. miRNA-targets related to autophagy initiation.

A, ULK1 complex

\begin{tabular}{|c|c|c|c|c|c|c|}
\hline miRNAs & $\begin{array}{c}\text { miRNA } \\
\text { expression }\end{array}$ & Targets & $\begin{array}{c}\text { Target } \\
\text { expression }\end{array}$ & $\begin{array}{c}\text { Autophagy } \\
\text { level }\end{array}$ & Cancer & $\begin{array}{c}\text { Chemotherapy } \\
\text { resistance }\end{array}$ \\
\hline $\mathrm{miR}-26 \mathrm{a} / \mathrm{b}$ & Up & ULK1 & Down & Down & $\mathrm{HCC}$ & Down \\
\hline miR-106a & Up & ULK1 & Down & Down & $\begin{array}{l}\text { Lung } \\
\text { adenocarcinoma }\end{array}$ & Down \\
\hline $\operatorname{miR}-25$ & Down & ULK1 & Up & Up & Breast cancer & Up \\
\hline miR-409-3p & Up & Fip200 & Down & Down & Ovarian cancer & Down \\
\hline
\end{tabular}

B, PI3K complex

\begin{tabular}{|c|c|c|c|c|c|c|}
\hline miRNAs & $\begin{array}{c}\text { miRNA } \\
\text { expression }\end{array}$ & Targets & $\begin{array}{c}\text { Target } \\
\text { expression }\end{array}$ & $\begin{array}{c}\text { Autophagy } \\
\text { level }\end{array}$ & Cancer & $\begin{array}{c}\text { Chemotherapy } \\
\text { resistance }\end{array}$ \\
\hline miR-140-5p & Up & ATG14 & Down & Down & Myeloma & Down \\
\hline $\operatorname{miR}-152$ & Up & ATG14 & Down & Down & Ovarian cancer & Down \\
\hline miR-30a & Up & Beclin-1 & Down & Down & Osteosarcoma & Down \\
\hline miR-30d & Down & Beclin-1 & Up & $\mathrm{Up}$ & UTC & Up \\
\hline $\operatorname{miR}-216 b$ & Up & $\begin{array}{l}\text { Beclin-1, } \\
\text { UVRAG and } \\
\text { ATG5 }\end{array}$ & Down & Down & Melanoma & Down \\
\hline $\operatorname{miR}-409-3 p$ & Up & Beclin-1 & Down & Down & Colon cancer & Down \\
\hline $\operatorname{miR}-216 b$ & Up & Beclin-1 & Down & Down & NSCLC & Down \\
\hline
\end{tabular}

C, mTOR and mTOR pathway

\begin{tabular}{llllll}
\hline miRNAs & $\begin{array}{c}\text { miRNA } \\
\text { expression }\end{array}$ & Targets & $\begin{array}{c}\text { Targets } \\
\text { expression }\end{array}$ & $\begin{array}{c}\text { Autophagy } \\
\text { level }\end{array}$ & $\begin{array}{c}\text { Chemotherapy } \\
\text { resistance }\end{array}$ \\
\hline miR-338-3p & $\mathrm{Up}$ & mTOR & Down & Up & Colon cancer \\
miR-193-5p & $\mathrm{Up}$ & mTOR & Down & Up & Glioma \\
miR-155 & $\mathrm{Up}$ & PTEN & Down & Down & Osteosarcoma \\
miR-21 & Down & PTEN & Up & Up & Breast cancer \\
miR-21 & $\mathrm{Up}$ & PTEN & Down & Down & HCC \\
miR-142-3p & $\mathrm{Up}$ & HMGB1 & Down & Up & NSCLC \\
miR-410-3p & $\mathrm{Up}$ & HMGB1 & Down & Up & PDAC \\
miR-218 & $\mathrm{Up}$ & HMGB1 & Down & Up & Endometrial \\
miR-22 & $\mathrm{Up}$ & HMGB1 & Down & Up & Oncer \\
miR-34a & $\mathrm{Up}$ & HMGB1 & Down & Up & Retinoblastoma
\end{tabular}

Up, upregulated; Down, downregulated; HCC, hepatocellular carcinoma; UTC, undifferentiated thyroid carcinoma; NSCLC, non-small cell lung cancer; PDAC, pancreatic ductal adenocarcinoma; ULK1, UNC-51-like autophagy-activating kinase 1; FIP20, focal adhesion kinase family interacting protein of $200 \mathrm{kDa}$; ATG, autophagy-related gene; UVRAG, UV resistance-associated gene; PTEN, phosphatase and tension homolog; HMGB1, high-mobility group box 1.

breast cancer cell growth and upregulated miRNA-375; the overexpression of miR-375 decreased the expression of its target $A T G 7$, which in turn mediated autophagy, which is beneficial in preventing fulvestrant resistance (51-56). Apigenin was found to significantly increase adriamycin sensitivity, induce the overexpression of miR-520b, and inhibit $A T G 7$-related autophagy in BEL-7402/ADM cells; these results were verified in a nude mouse xenograft as well as in vitro (52). The expression level of lncRNA-XIST (an oncogene in colorectal cancer) was found to be significantly increased in cisplatin-resistant A549 cells. Hence, IncRNA-turbulence/miR-17/autophagy may be a target for improving chemotherapy resistance in NSCLC 
Table II. miRNA-targets related to autophagosome formation.

A, ATG12-ATG5-ATG16L complex

\begin{tabular}{|c|c|c|c|c|c|c|}
\hline miRNAs & $\begin{array}{c}\text { miRNA } \\
\text { expression }\end{array}$ & Targets & $\begin{array}{c}\text { Target } \\
\text { expression }\end{array}$ & $\begin{array}{c}\text { Autophagy } \\
\text { level }\end{array}$ & Cancer & $\begin{array}{c}\text { Chemotherapy } \\
\text { resistance }\end{array}$ \\
\hline miR-23b-3p & Down & ATG12 & Up & Up & Gastric cancer & Up \\
\hline $\operatorname{miR}-410$ & Up & ATG16L & Down & Down & Osteosarcoma & Down \\
\hline miR-181a & Up & ATG5 & Down & Down & Gastric cancer & Down \\
\hline miR-142-3p & $\mathrm{Up}$ & ATG5/ATG16L & Down & Down & $\mathrm{HCC}$ & Down \\
\hline $\operatorname{miR}-23 b-3 p$ & $\mathrm{Up}$ & ATG12 & Down & Down & Gastric cancer & Down \\
\hline
\end{tabular}

B, LC3B complex

\begin{tabular}{|c|c|c|c|c|c|c|}
\hline miRNAs & $\begin{array}{c}\text { miRNA } \\
\text { expression }\end{array}$ & Targets & $\begin{array}{c}\text { Target } \\
\text { expression }\end{array}$ & $\begin{array}{c}\text { Autophagy } \\
\text { level }\end{array}$ & Cancer & $\begin{array}{c}\text { Chemotherapy } \\
\text { resistance }\end{array}$ \\
\hline miR-34a & Up & ATG4B & Down & Down & Prostate cancer & Down \\
\hline $\operatorname{miR}-34 c-5 p$ & $\mathrm{Up}$ & ATG4B & Down & Down & Cervical cancer & Down \\
\hline miR-375 & Up & ATG7 & Down & Down & Breast cancer & Down \\
\hline miR-520b & Up & ATG7 & Down & Down & $\mathrm{HCC}$ & Down \\
\hline miR-17 & Up & ATG7 & Down & Down & NSCLC & Down \\
\hline $\operatorname{miR}-423-5 p$ & $\mathrm{Up}$ & ATG7 & Down & Down & $\mathrm{HCC}$ & Down \\
\hline miR-17 & Down & ATG7 & Up & Up & Glioblastoma & Up \\
\hline miR-119a-5p & Up & ATG7 & Down & Down & $\mathrm{HCC}$ & Down \\
\hline
\end{tabular}

Up, upregulated; Down, downregulated; HCC, hepatocellular carcinoma; NSCLC, non-small cell lung cancer; ATG, autophagy-related gene; LC3B, microtubule-associated protein light chain 3.

patients by the knockdown of lncRNA-XIST to regulate miR-7 expression and inhibit $A T G 7$ expression so as to restore the sensitivity of cisplatin-resistant A549 cells (53). miR-423-5p is inactivated by ceramide. A previous study reported that miR-423-5p downregulates the expression of its target ATG7, leading to autophagy inhibition. In addition, miR-423-5p was found to downregulate the expression of $E R K$ to inhibit the proliferation of cells. Altogether, these results in a previous study implied that miR-423-5p induced the inhibition of autophagy and cell proliferation increased the apoptosis of cells and improved the sensitivity of HCC cells to sorafenib (54). miR-17 was demonstrated to negatively regulate $A T G 7$, and the downregulation of miR-17 was found to increase the expression of ATG7. miR-17 also enhanced autophagy, drug sensitivity of temozolomide, and the sensitivity of glioblastoma to low-dose ionizing radiation (55). Moreover, miR-199a-5p was found to specifically inhibit the expression of $A T G 7$, reduce autophagy, and enhance the inhibitory effect of cisplatin in regards to the proliferation of HCC cells (56).

miRNA-mediated autolysosome maturation changes the pattern of drug resistance in tumors. The development from autophagosome to autolysosome involves a complex system of new lysosomes and several large molecules that affect this process by altering the lysosomal activity. Lysosome-associated protein transmembrane $4 \beta$ (LAPTM4B) is one such molecule whose knockdown not only increases the lysosomal $\mathrm{pH}$ and inhibits lysosome and autophagosome fusion (57) but also causes lysosomal membrane permeabilization (58). LAPTM4B was reported as the target of miR-489 and was downregulated by $\mathrm{miR}-489$. Overexpression of $\mathrm{miR}-489$ was found to induce LAPTM4B-related autophagy and inhibit drug resistance in breast cancer cells (59). LAMP-1 and LAMP-2 are proteins that play a vital role in lysosomal biogenesis (60). miR-487-5p targets LAMP2, and knockdown of miR-487-5p was found to markedly increase levels of LAMP-2, enhance autophagy, and decrease cellular proliferation, whereas, miR-487b-5p suppression promoted temozolomide (TMZ) resistance in lung cancer cells (61).

\section{Conclusion and future directions}

The regulation of autophagy by non-coding miRNAs has been extensively researched in the last couple of years. Although a change in chemotherapy resistance for a single tumor may need the involvement of several different types of miRNAs, a single miRNA is capable of imparting chemotherapy resistance in a wide variety of tumors. Thus, resistance to a tumor chemotherapy drug can develop through various regulatory mechanisms of miRNAs via different target genes at every stage of autophagy. However, there are limitations to the studies on miRNAs and the involvement of autophagy in drug resistance. On one hand, some studies have focused on the relationship between an miRNA and its target genes related to autophagy at the molecular level through in vitro experiments. In addition, other studies have performed both 
in vitro experiments as well as animal models. In some studies, the experimental verification was confined to tumor transplantation, but not to human tissues. On the other hand, when multiple miRNAs are involved in the autophagy process of tumorigenesis, it is important to explore the specificity of the miRNA(s) or the specific steps(s) involved by using a large group of studies. miRNAs are thus regulated by lncRNAs or other upstream factors, making the regulation process extremely complex, which necessitates further research on the elaborate mechanisms involved in this process. Further insight into the exciting and complex mechanisms of miRNA-regulated autophagy is expected to be valuable in a variety of therapeutic applications, including the chemotherapy resistance of various types of tumors.

\section{Acknowledgements}

We thank all the individuals who assisted in this research.

\section{Funding}

No funding was received.

\section{Availability of data and materials}

All information included in this Review was supported by relevant references.

\section{Authors' contributions}

YH reviewed and edited the manuscript. TH prepared the original draft and completed the manuscript. ZX and LC designed the figures and YL completed the document retrieval. All authors read and approved the final manuscript.

\section{Ethics approval and consent to participate}

Not applicable.

\section{Patient consent for publication}

Not applicable.

\section{Competing interests}

The authors declare that they have no competing interests.

\section{References}

1. Konieczkowski DJ, Johannessen CM and Garraway LA: A convergence-based framework for cancer drug resistance. Cancer Cell 33: 801-815, 2018.

2. An X, Sarmiento C, Tan T and Zhu H: Regulation of multidrug resistance by microRNAs in anti-cancer therapy. Acta Pharm Sin B 7: 38-51, 2017.

3. Baek D, Villén J, Shin C, Camargo FD, Gygi SP and Bartel DP: The impact of microRNAs on protein output. Nature 455: 64-71, 2008.

4. Rupaimoole R and Slack FJ: MicroRNA therapeutics: Towards a new era for the management of cancer and other diseases. Nat Rev Drug Discov 16: 203-222, 2017.

5. Pan ST, Li ZL, He ZX, Qiu JX and Zhou SF: Molecular mechanisms for tumour resistance to chemotherapy. Clin Exp Pharmacol Physiol 43: 723-737, 2016.
6. Levy JMM, Towers CG and Thorburn A: Targeting autophagy in cancer. Nat Rev Cancer 17: 528-542, 2017.

7. Tooze SA and Dikic I: Autophagy captures the nobel prize. Cell 167: 1433-1435, 2016

8. Kimmelman AC and White E: Autophagy and tumor metabolism. Cell Metab 25: 1037-1043, 2017.

9. Mizushima N and Komatsu M: Autophagy: Renovation of cells and tissues. Cell 147: 728-741, 2011.

10. Glick D, Barth S and Macleod KF: Autophagy: Cellular and molecular mechanisms. J Pathol 221: 3-12, 2010.

11. Yu L, Chen Y and Tooze SA: Autophagy pathway: Cellular and molecular mechanisms. Autophagy 14: 207-215, 2018.

12. Hurley JH and Young LN: Mechanisms of autophagy initiation. Annu Rev Biochem 86: 225-244, 2017.

13. Jin F, Wang Y, Li M, Zhu Y, Liang H, Wang C, Wang F, Zhang CY, Zen K and Li L: miR-26 enhances chemosensitivity and promotes apoptosis of hepatocellular carcinoma cells through inhibiting autophagy. Cell Death Dis 8: e2540, 2017.

14. Rothschild SI, Gautschi O, Batliner J, Gugger M, Fey MF and Tschan MP: MicroRNA-106a targets autophagy and enhances sensitivity of lung cancer cells to Src inhibitors. Lung Cancer 107: 73-83, 2017.

15. Wang Z, Wang N, Liu P, Chen Q, Situ H, Xie T, Zhang J, Peng C, Lin Y and Chen J: MicroRNA-25 regulates chemoresistance-associated autophagy in breast cancer cells, a process modulated by the natural autophagy inducer isoliquiritigenin. Oncotarget 5: 7013-7026, 2014.

16. Hara T, Takamura A, Kishi C, Iemura S, Natsume T, Guan JL and Mizushima N: FIP200, a ULK-interacting protein, is required for autophagosome formation in mammalian cells. J Cell Biol 181: 497-510, 2008.

17. Cheng Y, Ban R, Liu W, Wang H, Li S, Yue Z, Zhu G, Zhuan Y and Wang C: miRNA-409-3p enhances cisplatin-sensitivity of ovarian cancer cells by blocking the autophagy mediated by Fip200. Oncol Res, Jan 2, 2018 (Epub ahead of print).

18. Lu D, Yang C, Zhang Z, Cong Y and Xiao M: Knockdown of Linc00515 inhibits multiple myeloma autophagy and chemoresistance by upregulating miR-140-5p and downregulating ATG14. Cell Physiol Biochem 48: 2517-2527, 2018.

19. Marchese FP, Raimondi I and Huarte M: The multidimensional mechanisms of long noncoding RNA function. Genome Biol 18: 206, 2017.

20. Yoon JH, Abdelmohsen $\mathrm{K}$ and Gorospe M: Functional interactions among microRNAs and long noncoding RNAs. Semin Cell Dev Biol 34: 9-14, 2014.

21. He J, Yu JJ, Xu Q, Wang L, Zheng JZ, Liu LZ and Jiang BH: Downregulation of ATG14 by EGR1-MIR152 sensitizes ovarian cancer cells to cisplatin-induced apoptosis by inhibiting cyto-protective autophagy. Autophagy 11: 373-384, 2015 .

22. Xu R, Liu S, Chen H and Lao L: MicroRNA-30a downregulation contributes to chemoresistance of osteosarcoma cells through activating Beclin-1-mediated autophagy. Oncol Rep 35: 1757-1763, 2016.

23. Zheng B, Zhu H, Gu D, Pan X, Qian L, Xue B, Yang D, Zhou J and Shan Y: MiRNA-30a-mediated autophagy inhibition sensitizes renal cell carcinoma cells to sorafenib. Biochem Biophys Res Commun 459: 234-239, 2015.

24. Zhang Y, Yang WQ, Zhu H, Qian YY, Zhou L, Ren YJ, Ren XC, Zhang L, Liu XP, Liu CG, et al: Regulation of autophagy by miR-30d impacts sensitivity of anaplastic thyroid carcinoma to cisplatin. Biochem Pharmacol 87: 562-570, 2014.

25. Luo M, Wu L, Zhang K, Wang H, Wu S, O'Connell D, Gao T, Zhong $\mathrm{H}$ and Yang Y: miR-216b enhances the efficacy of vemurafenib by targeting Beclin-1, UVRAG and ATG5 in melanoma. Cell Signal 42: 30-43, 2018.

26. Tan S, Shi H, Ba M, Lin S, Tang H, Zeng $X$ and Zhang X: miR-409-3p sensitizes colon cancer cells to oxaliplatin by inhibiting Beclin-1-mediated autophagy. Int J Mol Med 37: 1030-1038, 2016.

27. Chen K and Shi W: Autophagy regulates resistance of non-small cell lung cancer cells to paclitaxel. Tumour Biol 37: 10539-10544, 2016.

28. Huang J, Ni J, Liu K, Yu Y, Xie M, Kang R, Vernon P, Cao L and Tang D: HMGB1 promotes drug resistance in osteosarcoma. Cancer Res 72: 230-238, 2012.

29. Chen Y, Zhou X, Qiao J and Bao A: MiR-142-3p overexpression increases chemo-sensitivity of NSCLC by inhibiting HMGB1-mediated autophagy. Cell Physiol Biochem 41: 1370-1382, 2017 
30. Xiong J, Wang D, Wei A, Ke N, Wang Y, Tang J, He S, Hu W and Liu X: MicroRNA-410-3p attenuates gemcitabine resistance in pancreatic ductal adenocarcinoma by inhibiting HMGB1-mediated autophagy. Oncotarget 8: 107500-107512, 2017.

31. Ran X, Yang J, Liu C, Zhou P, Xiao L and Zhang K: miR-218 inhibits HMGB1-mediated autophagy in endometrial carcinoma cells during chemotherapy. Int J Clin Exp Pathol 8: 6617-6626, 2015.

32. Guo S, Bai R, Liu W, Zhao A, Zhao Z, Wang Y, Wang Y, Zhao W and Wang W: miR-22 inhibits osteosarcoma cell proliferation and migration by targeting HMGB1 and inhibiting HMGB1-mediated autophagy. Tumour Biol 35: 7025-7034, 2014.

33. Liu K, Huang J, Xie M, Yu Y, Zhu S, Kang R, Cao L, Tang D and Duan X: MIR34A regulates autophagy and apoptosis by targeting HMGB1 in the retinoblastoma cell. Autophagy 10 : $442-452,2014$

34. Kim YC and Guan KL: mTOR: A pharmacologic target for autophagy regulation. J Clin Invest 125: 25-32, 2015.

35. Han J, Li J, Tang K, Zhang H, Guo B, Hou N and Huang C: miR-338-3p confers 5-fluorouracil resistance in p53 mutant colon cancer cells by targeting the mammalian target of rapamycin. Exp Cell Res 360: 328-336, 2017.

36. Jiang C, Shen F, Du J, Fang X, Li X, Su J, Wang X, Huang X and Liu Z: Upregulation of CASC2 sensitized glioma to temozolomide cytotoxicity through autophagy inhibition by sponging miR-193a-5p and regulating mTOR expression. Biomed Pharmacother 97: 844-850, 2018.

37. Hildebrandt MA, Yang H, Hung MC, Izzo JG, Huang M, Lin J, Ajani JA and Wu X: Genetic variations in the $\mathrm{PI} 3 \mathrm{~K} / \mathrm{PTEN} / \mathrm{AKT} / \mathrm{mTOR}$ pathway are associated with clinical outcomes in esophageal cancer patients treated with chemoradiotherapy. J Clin Oncol 27: 857-871, 2009.

38. Lim HJ, Crowe P and Yang JL: Current clinical regulation of $\mathrm{PI} 3 \mathrm{~K} / \mathrm{PTEN} / \mathrm{Akt} / \mathrm{mTOR}$ signalling in treatment of human cancer J Cancer Res Clin Oncol 141: 671-689, 2015.

39. Wang L, Tang B, Han H, Mao D, Chen J, Zeng Y and Xiong M: miR-155 affects osteosarcoma MG-63 cell autophagy induced by adriamycin through regulating PTEN-PI3K/AKT/mTOR signaling pathway. Cancer Biother Radiopharm 33: 32-38, 2018.

40. Yu X, Li R, Shi W, Jiang T, Wang Y, Li C and Qu X: Silencing of MicroRNA-21 confers the sensitivity to tamoxifen and fulvestrant by enhancing autophagic cell death through inhibition of the PI3K-AKT-mTOR pathway in breast cancer cells. Biomed Pharmacother 77: 37-44, 2016

41. He C, Dong X, Zhai B, Jiang X, Dong D, Li B, Jiang H, Xu S and Sun X: MiR-21 mediates sorafenib resistance of hepatocellular carcinoma cells by inhibiting autophagy via the PTEN/Akt pathway. Oncotarget 6: 28867-28881, 2015.

42. Mizushima N: Autophagy: Process and function. Genes Dev 21: 2861-2873, 2007.

43. Kirkin V, McEwan DG, Novak I and Dikic I: A role for ubiquitin in selective autophagy. Mol Cell 34: 259-269, 2009.

44. YiRen H, YingCong Y, Sunwu Y, Keqin L, Xiaochun T, Senrui C, Ende C, XiZhou L and Yanfan C: Long noncoding RNA MALAT1 regulates autophagy associated chemoresistance via miR-23b-3p sequestration in gastric cancer. Mol Cancer 16 174, 2017.

45. Chen R, Li X, He B and Hu W: MicroRNA-410 regulates autophagy-related gene ATG16L1 expression and enhances chemosensitivity via autophagy inhibition in osteosarcoma. Mol Med Rep 15: 1326-1334, 2017.
46. Zhao J, Nie Y, Wang H and Lin Y: MiR-181a suppresses autophagy and sensitizes gastric cancer cells to cisplatin. Gene 576: 828-833, 2016.

47. Zhang K, Chen J, Zhou H, Chen Y, Zhi Y, Zhang B, Chen L, Chu X, Wang R and Zhang C: PU.1/microRNA-142-3p targets ATG5/ATG16L1 to inactivate autophagy and sensitize hepatocellular carcinoma cells to sorafenib. Cell Death Dis 9: 312, 2018.

48. An Y, Zhang Z, Shang Y, Jiang X, Dong J, Yu P, Nie Y and Zhao Q: miR-23b-3p regulates the chemoresistance of gastric cancer cells by targeting ATG12 and HMGB2. Cell Death Dis 6: e1766, 2015.

49. Liao H, Xiao Y, Hu Y, Xiao Y, Yin Z, Liu L, Kang X and Chen Y: Methylation-induced silencing of miR-34a enhances chemoresistance by directly upregulating ATG4B-induced autophagy through AMPK/mTOR pathway in prostate cancer. Oncol Rep 35: 64-72, 2016.

50. Wu Y, Ni Z, Yan X, Dai X, Hu C, Zheng Y, He F and Lian J: Targeting the MIR34C-5p-ATG4B-autophagy axis enhances the sensitivity of cervical cancer cells to pirarubicin. Autophagy 12: 1105-1117, 2016.

51. Liu L, Shen W, Zhu Z, Lin J, Fang Q, Ruan Y and Zhao H: Combined inhibition of EGFR and c-ABL suppresses the growth of fulvestrant-resistant breast cancer cells through miR-375-autophagy axis. Biochem Biophys Res Commun 498: 559-565, 2018.

52. Gao AM, Zhang XY, Hu JN and Ke ZP: Apigenin sensitizes hepatocellular carcinoma cells to doxorubic through regulating miR-520b/ATG7 axis. Chem Biol Interact 280: 45-50, 2018

53. Sun W, Zu Y, Fu X and Deng Y: Knockdown of lncRNA-XIST enhances the chemosensitivity of NSCLC cells via suppression of autophagy. Oncol Rep 38: 3347-3354, 2017.

54. Stiuso P, Potenza N, Lombardi A, Ferrandino I, Monaco A, Zappavigna S, Vanacore D, Mosca N, Castiello F, Porto S, et al: MicroRNA-423-5p promotes autophagy in cancer cells and is increased in serum from hepatocarcinoma patients treated with sorafenib. Mol Ther Nucleic Acids 4: e233, 2015.

55. Comincini S, Allavena G, Palumbo S, Morini M, Durando F, Angeletti F, Pirtoli L and Miracco C: MicroRNA-17 regulates the expression of ATG7 and modulates the autophagy process, improving the sensitivity to temozolomide and low-dose ionizing radiation treatments in human glioblastoma cells. Cancer Biol Ther 14: 574-586, 2013.

56. Xu N, Zhang J, Shen C, Luo Y, Xia L, Xue F and Xia Q Cisplatin-induced downregulation of miR-199a-5p increases drug resistance by activating autophagy in HCC cell. Biochem Biophys Res Commun 423: 826-831, 2012.

57. Li Y, Iglehart JD, Richardson AL and Wang ZC: The amplified cancer gene LAPTM4B promotes tumor growth and tolerance to stress through the induction of autophagy. Autophagy 8: 273-274, 2012.

58. Blom T, Li S, Dichlberger A, Bäck N, Kim YA, LoizidesMangold U, Riezman H, Bittman R and Ikonen E: LAPTM4B facilitates late endosomal ceramide export to control cell death pathways. Nat Chem Biol 11: 799-806, 2015.

59. Soni M, Patel Y, Markoutsa E, Jie C, Liu S, Xu P and Chen H: Autophagy, cell viability, and chemoresistance are regulated by miR-489 in breast cancer. Mol Cancer Res 16: 1348-1360, 2018.

60. Eskelinen EL: Roles of LAMP-1 and LAMP-2 in lysosome biogenesis and autophagy. Mol Aspects Med 27: 495-502, 2006.

61. Bao L, Lv L, Feng J, Chen Y, Wang X, Han S and Zhao H: miR-487b-5p regulates temozolomide resistance of lung cancer cells through LAMP2-medicated autophagy. DNA Cell Biol 35: $385-392,2016$ 